\title{
Predictability of eplet mismatch to acute rejection in low level HLA mismatched kidney transplantation: validation analysis of Korean Organ Transplantation Registry (KOTRY) data
} \author{
Yun Ji Hong ${ }^{7}$, Borae G. Park ${ }^{8}$ \\ 'Division of Nephrology, Department of Internal Medicine, Seoul National University Bundang Hospital, Seongnam, Korea \\ ${ }^{2}$ Department of Surgery, Gachon University Gil Medical Center, Incheon, Korea \\ ${ }^{3}$ Division of Nephrology, Department of Internal Medicine, Ajou University School of Medicine, Suwon, Korea \\ ${ }^{4}$ Department of Surgery, Sungkyunkwan University School of Medicine, Suwon, Korea \\ ${ }^{5}$ Department of Internal Medicine, Seoul National University College of Medicine, Seoul, Korea \\ ${ }^{6}$ Department of Surgery, Seoul National University Hospital, Seoul, Korea \\ ${ }^{7}$ Department of Laboratory Medicine, Seoul National University Bundang Hospital, Seongnam, Korea \\ ${ }^{8}$ Department of Laboratory Medicine, Korea University Guro Hospital, Seoul, Korea
}

Jong Cheol Jeong ${ }^{1}$, Hyeong Eun Son ${ }^{1}$, Yeon Ho Park ${ }^{2}$, Inwhee Park ${ }^{3}$, Jae Berm Park ${ }^{4}$, Dong Wan Chae ${ }^{5}$, Jaeseok Yang ${ }^{6}$,

Background: Epitope matching has been shown to better predict allograft survival and development of de novo donor-specific antibodies. There was no large scale Korean study for epitope matching and kidney transplantation outcomes.

Methods: Patients included in the Korean Organ Transplantation Registry (KOTRY) were used. Kidney transplant recipients who received transplants from 2014 to 2017 were enrolled. As an external validation cohort, retrospective KOTRY data (kidney transplant pairs from 2009 to 2012) were used. Human leucocyte antigen (HLA) four-digit genotypes were imputed by matching to the four-digit haplotype distribution in the Korean bone marrow donor program. DQ genotyping was imputed by linkage-disequilibrium association. The primary outcome measurement was biopsy-proven acute rejection.

Results: Among 5,872 donor-recipient pairs, four-digit haplotype pairs were successfully imputed. Mean numbers of mismatched class I and class II eplets were 10.5 \pm 6.8 (range, 0-35) and 24.1 \pm 17.4 (range, 0-85), respectively. Mean eplet mismatch numbers were increased according to the number of HLA antigen mismatches (beta, 9.340; 95\% confidence interval [Cl], 9.1459.534; $\mathrm{P}<0.001$ ). Eplet mismatch was associated with acute rejection in the unadjusted models (class I: $1.022,95 \% \mathrm{Cl}, 1.011-1.033$; $\mathrm{P}<0.001$; class II: $1.011,95 \% \mathrm{Cl}, 1.007-1.015 ; \mathrm{P}<0.001)$. When controlled with clinical covariates and HLA mismatch numbers, eplet mismatch numbers did not show additional predictability for acute rejection (AUC comparison: eplet model vs. HLA serotype model, 0.615 [95\% Cl, 0.594-0.636] vs. 0.619 [95\% Cl, 0.598-0.640]; P=0.491). However, eplet class II mismatches over 60 were shown to be significant risk predictors in low HLA serotype mismatches (one or two mismatches) in a non-linear model. Conclusions: In this external validation study, eplet mismatches in class II MHC were found to be significant risk factors for acute rejection with a low number of HLA mismatches (one or two mismatches).

Corresponding author: Jong Cheol Jeong

E-mail: jcj0425@empal.com

\section{() The Korean Society for Transplantation}

This is an Open Access article distributed under the terms of the Creative Commons Attribution Non-Commercial License (http://creativecommons.org/licenses/by-nc/4.0/) which permits unrestricted non-commercial use, distribution, and reproduction in any medium, provided the original work is properly cited. 